\title{
Sleep telemedicine: a glance into the future
}

\begin{abstract}
Sleep breathing disorders have characteristic symptoms related with snoring and apneas during sleep. There is much comorbidity associated with bad prognosis in these patients. The treatment with CPAP and the adherence to the device is very important for the prognosis and increasing compliance of the use of positive airway pressure (PAP) is the greatest challenge in the treatment of sleep disorders.
\end{abstract}

Telemedicine is rapidly expanding technology involving the exchange of medical information to assist diagnosis and treatment at a distance. Actually, the use of telemedicine has been reported as a way to supervision to raise the adherence in treatment with CPAP. Basically it implies the distribution of health services by healthcare providers using information and communication technologies (ICT) to exchange at distance information useful for diagnosis, education and monitoring of diseases (eHealth). Generally, telehealth interventions include: video or telephone links with healthcare professionals in real time or using store-and-forward technologies, internet-based telecommunication systems with healthcare professionals, wired and wireless telemonitoring of physiological parameters such as spirometry, respiratory rate, blood pressure or oxygen saturations processed or authorised by a healthcare professional with feedback to the patient and even telemonitoring of patients on home mechanical ventilation. This review wants to summarize some of the aspects and benefit of telemedicine in Sleep Breathing disorders.

Keywords: sleep, telemedicine, technologies, video communications, health care
Volume I Issue 3- 2017

\section{Marco Solis, Patricia Maggio, Manuel Ibarrola, Silvia Quadrelli}

Sanatorio Güemes University Hospital, Buenos Aires, Argentina

Correspondence: Marco Solis, Sanatorio Güemes University Hospital, Buenos Aires, Argentina, Tel 00549I160003392, Emailmsolisaramayo@yahoo.com.ar

Received: September 28, 2017 | Published: October 27, 2017
Abbrevations: PAP, positive airway pressure; ICT, information and communication technologies; OSAS, obstructive sleep apnea syndrome; CPAP, continuous positive airway pressure; HSAT, home sleep apnea test; CVT, clinical video telehealth

\section{Perspective}

Obstructive sleep apnea syndrome (OSAS) is associated with significant morbidity, including increased risk for car accidents, hypertension, metabolic diseases and impaired cognition..$^{1,2}$ Persons with OSAS have been found to have increased use health care resources when compared with controls, ${ }^{3}$ and the detection of OSAS and subsequent treatment with nocturnal continuous positive airway pressure (CPAP) can significantly improve quality of life and reduce mortality. ${ }^{4}$ Increasing compliance with the use of positive airway pressure (PAP) is the greatest challenge in the treatment of sleep disorders. Most of educational approaches tested to improve adherence have had only modest impact on compliance and only the intensive support protocols have had greater impact. However, these intensive supports imply an important amount of time and resources to be implemented. In most of the experienced sleep units, usual care of patients initiating PAP treatment includes the initial titration of the CPAP device to the right pressure, a visit to the clinic or phone call to check on the patient's comfort and the correct use of the devices within one week of CPAP initiation at home, and then regular in-office visits. However, many physicians and sleep units cannot maintain that level of care even with remote patients, and also patients' self-reported difficulties with CPAP or subjective compliance do not always provide reliable information to guide appropriate clinical management of OSA in these care conditions. ${ }^{5}$
The use of telemedicine has been reported in a variety of forms since the 1960. Telemedicine has been defined in a variety of ways. Basically it implies the distribution of health services by healthcare providers using information and communication technologies (ICT) to exchange at distance information useful for diagnosis, education and monitoring of diseases (eHealth). ${ }^{6}$ There is no a unique definition but it is generally accepted that includes use of cable connections, radio, optical means or other electromagnetic channels to transmit or receive signals, such as voice, data or video communications. Generally, telehealth interventions, ${ }^{7}$ include: video or telephone links with healthcare professionals in real time or using store-andforward technologies, internet-based telecommunication systems with healthcare professionals, wired and wireless telemonitoring of physiological parameters such as spirometry, respiratory rate, blood pressure or oxygen saturations processed or authorised by a healthcare professional with feedback to the patient ${ }^{8}$ and even telemonitoring of patients on home mechanical ventilation. Recent applications of telemedicine permit remote consultations in specialities from dermatology to psychiatry, the transmission of electrocardiograms and radiological images, the provision of accident and emergency expertise during accidents, remote fetal monitoring, and very importantly, education and second for health professionals. The rapid developments in the technology are giving health care organisations to find the way of approaching populations far from their physical buildings or with difficulties for transportation. It may also permit new ways of providing health care and better control of home care, changing the traditional roles of health care professionals. The potential benefits of telemedicine include improved access to health care, reduced waiting time for appointments, and increased adherence 
to treatment plans. ${ }^{9}$ Because many sleep disorders, particularly sleep apnea, are chronic conditions, and require a continuous treatment and monitoring of therapy success, ICT and new information technologies could be useful to establish diagnostic and therapeutic strategies. Installing cost-efficient technologies for an initial simple diagnosis, rapid treatment initiation, and a better and more efficient long-term monitoring of adherence and compliance, may help patients to get better outcomes in the control of their diseases. ${ }^{10}$ Telemedicine in sleep disordered breathing may be used with diagnostic purposes (telemonitored polysomnography, long-term polygraphic monitoring), for remote continuous positive airway pressure titration, to monitor CPAP adherence and compliance and even for patient counselling to reinforce therapy. Sleep telemedicine can be offered synchronously or asynchronously. Synchronous telemedicine implies real-time providerpatient interaction over the internet, telephone, or via clinical video telehealth (CVT), while asynchronous telemedicine is not a real-time interaction but is based on "store-and-forward" technologies, such as remote home sleep apnea test (HSAT) interpretation and positive airway pressure machine data review via cloud-based platforms such as Resmed AirView ${ }^{\mathrm{TM}}$ and Respironics EncoreAnywhere ${ }^{\mathrm{TM}} .^{11}$

CVT technology permits sessions that emulate face-to-face encounters with real-time audiovisual communication. Usually patients are accompanied by healthcare technicians, respiratory therapists, or nurses with some training in the use of the system. In one of the programmes described primary care physicians at originating sites refer patients with suspected sleep disorders to the distant site, from that central location, sleep physicians communicated with 3 groups of patients simultaneously through the use of CVT. ${ }^{12}$ CVT has also shown to be useful to teach patients about their PAP systems showing similar levels of knowledge $(93.6 \%$ vs. $92.1 \%$ of correct responses, $\mathrm{p}=0.935$ ) and practical skill performance (i.e. mask placement). ${ }^{13}$ CVT based programmes demonstrated to be effective in increasing adherence to PAP therapy with some studies disclosing a high level of patient satisfaction $(9.50 \pm 0.72 \text { over } 10)^{14}$ and similar adherence rates $(340.55 \mathrm{~min} / \mathrm{night}$ vs. $305.31 \mathrm{~min} /$ night, $\mathrm{p}=0.153$ ) measured by retrospective PAP machine..$^{10,14}$

Other technology used in the last years is the web-based programmes. Internet-based support for the first 30 days resulted in the same levels of PAP adherence and no difference in functional outcomes (measured by Functional Outcomes of Sleep Questionnaire) or patient satisfaction (measured by the Client Satisfaction Questionnaire) when compared to conventional in presence followup. Interestingly, now many PAP devices are equipped with wireless modems to provide adherence and efficacy monitoring. In those systems data are transmitted daily to the device company's internet cloud. A pilot study from the Veterans Affairs San Diego Healthcare System $(n=45)$ where each participant were provided with an AutoSet Spirit flow generator unit (ResMed Corp, Poway, CA) set to fixedmode pressure and all CPAP flow generators were outfitted with a ResTraxx wireless transmitter (ResMed Corp, Poway, CA) to allow the remote data collection of compliance and efficacy information. While the patient slept, data were collected at the next day, and became available on a sleep clinic's computer. The study confirmed the $100 \%$ accuracy rate of wireless data transmission in comparison to manually downloaded data and did not find a statistically significant effect for the telemedicine group on the primary outcome measure of CPAP compliance. ${ }^{15}$ sMore recently, several sleep-related smartphone apps have been developed. However, most of them do not provide site-to-site communication. A study using smartphones that allowed both to assess outcomes and to provide lifestyle counselling showed that CPAP treatment supported by this method of telemedicine alone did not significantly improve self-measured BP, CPAP adherence or physical activity when compared with standard care whereas sleepiness and quality of life scores significantly improved in both arms. ${ }^{16}$ In spite of negative initial results, this is an area in development and new apps are expected to be released in the next years. In general, the idea of telemedicine is well accepted by patients. Patients identified many practical barriers to in-person visits, including distance, cost and work interruptions and evidence shows that many of they are willing to consider video telemedicine as an option for their care. Some studies indicate that patients were equally satisfied with their provider and adherent to CPAP treatment whether they were seen in person or via video and that videoconferencing does not reduce patient satisfaction or treatment adherence. ${ }^{17}$ In regards to the rising costs of health care, telemedicine may be a promising solution, potentially resulting in cost-savings for patients and hospitals. In one economic review of video telemedicine, 22 of 36 studies reported this method to be more cost effective than standard office appointments. ${ }^{18}$ However, to evaluate the real cost/effectiveness of new methods of care such as telemedicine, a better definition of techniques, outcomes and comparison standards is required.

Telemedicine utilization is rising due to the increased availability and decreased cost of communication technology, in parallel with growing recognition of key areas of health care that may benefit from its use. However, further research is needed before generalising the results in some areas of medical practice to others. When considering the use of communication technologies, physicians must establish a true benefit that compensates the costs and efforts required to implement new technologies and the acquisition of new skills from health care providers and new habits from patients. Practitioners must be aware that the use of telemedicine technologies may require different clinical skills including new communication skills. ${ }^{19}$ The ways of giving information giving, the strategies to establish a human binding in spite of the distance and the techniques to assure the full comprehension of the information provided by these new methods are significantly different. The nature of the clinical encounter and the patient-doctor relationship changes radically and the common implicit understandings of traditional encounters should not be given for granted. There are some factors impeding the growth of telemedicine including the training for physicians and health-care providers that is time-consuming and the clinical encounter many be not as rewarding as the personal one, the confidence and malpractice issues, technical advances, cost effectiveness reimbursement and licensing. These issues may need to be considered and great care is needed to not reduce the clinical encounter to a mere recollection of information. Randomised controlled trials of telemedicine applications are feasible and we are in need of new studies applied to sleep disorders. The high speed of technology development will rapidly create new ways of using telemedicine in diagnosis and mainly monitoring of sleep disorder treatment and so, research about effectiveness, efficiency and appropriateness of telematics applications to health care are urgently needed to not waste a lot of money and energy in innovations that end in no clear benefit.

\section{Acknowledgements}

None. 


\section{Conflicts of interest}

The author declares no conflicts of interest.

\section{References}

1. Young T, Blustein J, Finn L, et al. Sleep-disordered breathing and motor vehicle accidents in a population-based sample of employed adults. Sleep. 1997;20(8):608-613.

2. Hedner J, Grote L. The link between sleep apnea and cardiovascular disease: time to target the nonsleepy sleep apneics? Am J Respir Crit Care Med. 2001;163(1):5-6.

3. Ronald J, Delaive K, Roos L, et al. Health care utilization in the 10 years prior to diagnosis in obstructive sleep Apnea syndrome patients. Sleep. 1999;22(2):225-229.

4. Engleman HM, Kingshott RN, Wraith PK, et al. Randomized placebocontrolled crossover trial of continuous positive airway pressure for mild sleep apnea/Hypopnea syndrome. Am J Respir Crit Care Med. 1999;159(2):461-467.

5. Verbraecken J. Telemedicine applications in sleep disordered breathing: thinking out of the box. Sleep Med Clin. 2016;11(4):445-459.

6. Ambrosino N, Vitacca M, Dreher M, et al. Tele-monitoring of ventilatordependent patients: a European respiratory society statement task force report ERS statement. Eur Respir J. 2016;48:648-663.

7. American Telemedicine Association. Tele-medicine glossary, USA.

8. Burgos F, Disdier C, Lopez de Santamaria E, et al. Telemedicine enhances quality of forced spirometry in primary care. Eur Respir J. 2012;39(6):1313-1318.

9. Kelly JM, Schwamm L, Bianchi M. Sleep telemedicine: a survey study of patient preferences. ISRN Neurol. 2012;2012:135329.
10. Coma-Del-Corral M, Alonso-Álvarez M, Allende M, et al. Reliability of telemedicine in the diagnosis and treatment of sleep apnea syndrome. Telemed J E Health. 2013;19(1):7-12.

11. Zia S, Fields BG. Sleep telemedicine: an emerging field's latest frontier. Chest. 2016;149(6):1556-1565.

12. Hirshkowitz M, Sharafkhaneh A. A telemedicine program for diagnosis and management of sleep-disordered breathing: the fast-track for sleep apnea tele-sleep program. Semin Respir Crit Care Med. 2014;35(5):560-570.

13. Isetta $\mathrm{V}$, León $\mathrm{C}$, Torres $\mathrm{M}$, et al. Telemedicine-based approach for obstructive sleep apnea management: building evidence. Interact $J$ Med Res. 2014;3(1):e6.

14. Parikh R, Touvelle M, Wang H, et al. Sleep telemedicine: patient satisfaction and treatment adherence. Telemed J E Health. 2011;17(8):609-614.

15. Stepnowsky CJ, Palau JJ, Marler MR, et al. Pilot randomized trial of the effect of wireless telemonitoring on compliance and treatment efficacy in obstructive sleep apnea. J Med Internet Res. 2007;9(2):e14.

16. Mendelson M, Vivodtzev I, Tamisier R, et al. CPAP treatment supported by telemedicine does not improve blood pressure in high cardiovascular risk OSA patients: a randomized, controlled trial. Sleep. 2014;37(11):18631870 .

17. Roshni P, Megan NT, Huaping W, et al. Telemedicine and e-Health. September. 2011;17(8):609-614.

18. Wade V, Karnon J, Elshaug A, et al. A systematic review of economic analyses of telehealth services using real time video communication. BMC Health Services Research. 2010;10:233.

19. Currell R, Urquhart C, Wainwright $P$, et al. Telemedicine versus face to face patient care: effects on professional practice and health care outcomes. Cochrane Database Syst Rev. 2000;2:CD002098. 